Annuaire suisse de politique de développement

18 | 1999

La Suisse et l'action humanitaire

\title{
Développement et urgence : le cas de l'OAP au Burundi
}

Daniel Fino

\section{(2) OpenEdition}

Édition électronique

URL : http://journals.openedition.org/aspd/674

DOI : 10.4000/aspd.674

ISSN : 1663-9669

Éditeur

Institut de hautes études internationales et du développement

\section{Édition imprimée}

Date de publication : 1 janvier 1999

Pagination : 73-82

ISSN : 1660-5934

\section{Référence électronique}

Daniel Fino, «Développement et urgence : le cas de l'OAP au Burundi », Annuaire suisse de politique de développement [En ligne], 18| 1999, mis en ligne le 12 juillet 2012, consulté le 07 septembre 2020. URL : http://journals.openedition.org/aspd/674; DOI : https://doi.org/10.4000/aspd.674 


\title{
DÉVELOPPEMENT ET URGENCE: LE CAS DE L'OAP AU BURUNDI
}

\author{
Daniel Fino*
}

\section{UN APPRENTISSAGE PAR LA PRATIQUE}

L'étude de cas de l'OAP (Opération d'appui à l'autopromotion) au Burundi montre qu'il est possible de soutenir le développement socio-économique à la base malgré une situation de crise et d'instabilité politico-militaire quasi permanente. La question qui se pose concrètement à un groupe d'opérateurs engagés dans la promotion du développement local ${ }^{2}$ est de savoir comment mettre en œuvre une démarche qui vise l'autopromotion des communautés de base dans un contexte caractérisé par des problèmes socio-économiques graves comme la perte des bases existentielles (habitation, travail, terres, infrastructures publiques...) et l'insécurité, dus à des affrontements entre différents groupes armés et, consécutivement, à des déplacements massifs de populations ${ }^{3}$ à l'intérieur de la province et du pays.

L'OAP est une organisation non gouvernementale avec un statut d'ONG internationale ${ }^{4}$. Elle s'est constituée en 1993 lorsqu'un projet d'aide bilatérale entre la Suisse et le Burundi ${ }^{5}$ est arrivé à terme. L'OAP s'est fixé comme but de soutenir les initiatives locales à la base dans les domaines de la production agricole, l'artisanat et le commerce. A peine quelques mois après son démarrage, l'OAP s'est vue confrontée aux graves troubles qui ont éclaté suite à l'assassinat du président Ndadayé, lorsque des milliers de personnes déplacées se sont installées dans les communes, soit chez l'habitant, soit en site protégé. Grâce au soutien financier (en moyenne 200'000 francs par an) de la Division de l'aide humanitaire de la DDC (Direction du développement et de la coopération), l'OAP a alors rapidement su intégrer la dimension d'aide d'urgence dans son

* Socio-économiste, chargé de cours à l'IUED et chargé du programme Burundi.

1. Cette contribution est une version modifiée de l'article de D. Fino, P. Kana et M. Ndikumwami «Articuler l'aide d'urgence avec l'aide au développement", Génocides et violences dans l'Afrique des Grands Lacs. Six propositions pour une réforme de la coopération internationale, Sonagral, Montpellier, 1997.

2. Le développement local est défini ici comme la volonté des différents acteurs présents dans une commune de changer la situation socio-économique des familles - et par conséquent de l'ensemble de la communauté. Il ne se fonde pas sur une hypothèse d'unanimité d'intérêts, mais suppose que le changement social dans une communauté relève premièrement de la responsabilité des acteurs locaux.

3. Dans la province de Bujumbura-Rural (actuellement composée de dix communes comptant une population d'environ 400'000 à 500'000 habitants), on pouvait recenser à certaines périodes (1993-1994) jusqu'à 50'000 personnes déplacées, dont seulement environ $10 \%$ se trouvaient dans des camps. La situation de crise provoquée par ces déplacements n'est pas comparable avec celle par exemple des réfugiés rwandais dans le Kivu où vivaient, entre 1995 et 1996, des centaines de milliers de réfugiés dans les camps. Notre analyse se réfère uniquement au cas des personnes déplacées au Burundi.

4. L'OAP représente l'IUED au Burundi. L'équipe des cadres est composée de quatre Burundaises (une sociopédagogue chargée de la direction, une économiste, une responsable de l'animation et une administratrice), provenant des deux ethnies principales.

5. Il s'agit du PADC (Programme d'appui au développement des communes d'Isale, Mubimbi et Kanyosha), présent dans la province de Bujumbura de 1982 à 1993. Le dernier responsable du PADC, l'ingénieur agronome J.-E. Beuret, a été ensuite le principal artisan de la nouvelle structure appelée OAP. Il a profondément marqué l'orientation donnée à l'OAP, et notamment la prise en compte de la dimension «urgence» dans une démarche d'autopromotion. 
programme de travail sans pour autant être en contradiction avec l'approche développement. Cela s'est traduit concrètement par son engagement pour

$\checkmark$ la coordination des secours afin d'harmoniser les modes d'intervention, en essayant notamment de coordonner les quantités et les types des aides à fournir (aides alimentaires, ustensiles de ménage, couvertures, savons, etc.) ;

๖ la médiation entre l'administration locale, les différents intervenants, la population résidente et les personnes déplacées ;

- la mobilisation des résidents et des personnes déplacées dans l'organisation de la distribution des aides.

Quelques semaines (parfois quelques jours) seulement après leur arrivée, les personnes déplacées ont exprimé le désir d'obtenir des houes et des semences pour cultiver. L'OAP a alors favorisé la négociation entre elles et les communes pour pouvoir utiliser des terres libres. Les houes ont été fournies gratuitement tandis que les semences ont été données sur crédit. Cette politique de diffusion de semences par des prêts s'est parfois heurtée à la distribution non concertée, par les agences d'aide alimentaire, de leur stock de nourriture.

Dès le début d'une situation de crise, l'OAP inclut dans sa démarche la population sédentaire qui doit supporter des charges importantes dans l'accueil, l'hébergement, le partage de la nourriture, la mise à disposition de terres, etc.

Très rapidement - et ce pratiquement dans toutes les communes - les personnes déplacées ont demandé à pouvoir développer des activités et pratiquer leur profession (maçon, cultivateur, menuisier...). Pour prendre toutes ces demandes en compte et ne pas créer d'injustices parmi les personnes déplacées ni entre ces dernières et les résidents, l'OAP a proposé de débattre de ces demandes en toute transparence entre les intéressés et d'impliquer tous les acteurs importants de la commune dans les décisions d'attribution des appuis (voir ci-après les commissions communales).

Dans les années qui suivirent, les personnes déplacées sont soit retournées dans leur commune d'origine, si la situation le permettait, soit parties dans une autre commune, ou se sont installées - avec le consentement des résidents - définitivement dans la commune de refuge.

L'OAP est aujourd'hui un programme qui favorise la recherche de solutions locales aux problèmes qui se posent aux communautés de base (personnes déplacées et résidents) dans trois domaines principalement:

- la relance d'initiatives économiques de particuliers et de groupements ;

口 la réhabilitation d'infrastructures publiques (écoles, dispensaires...);

口 la reconstruction de l'habitat ${ }^{6}$.

Il existe en outre un fonds spécial pour les secours (aide aux orphelins, aide alimentaire ponctuelle, aide d'urgence aux rapatriés, etc.).

6. Quelques précisions sur la démarche: l'OAP contribue à la reconstruction de l'habitat par un apport en tuiles, fenêtres, portes et briques. Ces matériaux sont confectionnés par les artisans de la région, créant ainsi des emplois et des revenus locaux. La même démarche est utilisée pour la réhabilitation d'infrastructures publiques (construction d'écoles, fabrication de bancs et pupitres, de lits de dispensaires, etc.). L'appui à des initiatives économiques concerne des crédits accordés à des groupes d'artisans, d'éleveurs, d'agriculteurs pour réaliser des petits projets productifs. 
Les premiers interlocuteurs de l'OAP sont les commissions communales, composées de représentants de tous les secteurs de la commune (administration, Eglise, groupes professionnels, association des femmes, comité des personnes déplacées...). Les appuis de l'OAP dans une commune dépendent largement du dynamisme et de l'engagement de la commission communale, qui joue un rôle central dans l'identification des projets, leur préparation, l'organisation du travail, le suivi et l'évaluation. Les relations entre l'OAP et les commissions communales sont de type contractuel. En l'absence de commissions communales - parce que les différents groupes n'ont pas pu s'entendre ou parce que l'aide se limite au soutien des activités économiques -, l'OAP travaille avec des associations relais qui, mis à part le fait qu'elles ne réunissent pas l'ensemble des acteurs, ont la même fonction que les commissions communales.

\section{PRINCIPES DE L'OAP}

Parmi les différents principes qui guident le travail de l'OAP, les trois suivants ont rencontré un certain intérêt auprès de la Division de l'aide humanitaire:

- L'OAP fait une analyse différenciée des personnes qu'elle considère comme en détresse et vulnérables et auxquelles l'aide d'urgence est nécessaire. Elle veille à ne pas sous-estimer la capacité des personnes déplacées de se prendre en charge ou au moins d'y contribuer et à limiter l'aide de type assistanat aux personnes qui ne peuvent effectivement pas s'en sortir par elles-mêmes. C'est pourquoi dans chaque situation elle doit systématiquement chercher à identifier cette capacité afin de «doser» l'aide de type assistanat en conséquence. Ces précautions permettent aux "victimes" de retrouver confiance en elles-mêmes, et ainsi la perspective d'une autonomie. On voit que dans cette optique, plutôt que d'appliquer une séquence d'aide d'urgence qui serait suivie par une séquence d'aide au développement, l'OAP dans la plupart des cas met en œuvre pratiquement dès le début une approche de type développement, avec, selon les cas, une composante d'aide d'urgence qu'il s'agit de gérer avec prudence.

• L'OAP aide les personnes déplacées à entrer le plus rapidement possible dans les circuits économiques habituels de l'agriculture, de l'artisanat, des services et du commerce. Cet axe de travail est le plus important dans le programme de ses activités. En effet, avoir une occupation rémunérée représente le meilleur moyen de sortir de la crise puisqu'elle permet aux familles de se nourrir, de contribuer à résoudre les problèmes sociaux de santé et éducation, et de garder leur dignité en (re)trouvant une position dans la société. En outre, le travail constitue une condition essentielle pour construire la paix.

- L'OAP a une position claire sur les rôles des acteurs:

- Le développement local (y compris la gestion de crises) relève de la responsabilité de tous les acteurs présents dans la commune; l'OAP se limite à favoriser la rencontre entre les différents acteurs, mais elle ne se substitue pas à eux pour la recherche de solutions.

- Les différents acteurs jouent des rôles complémentaires dans la société : l'administration publique a ses propres tâches bien spécifiques que les privés ou les associations ne peuvent accomplir, et vice-versa. Cette 
perspective nécessite un respect mutuel, une tolérance et une capacité d'écoute entre des acteurs qui ne poursuivent pas forcément les mêmes intérêts, et dans ce contexte l'OAP fait un travail de sensibilisation et de médiation.

- Dans des pays comme le Burundi, il y a un grand besoin d'organisations nationales non gouvernementales comme l'OAP qui s'engagent dans le développement local et l'aide d'urgence.

\section{QUELQUES ENSEIGNEMENTS TIRÉS DE L'EXPÉRIENCE DE L'OAP}

Cinq années d'expériences de l'OAP permettent de tirer quelques enseignements qui peuvent s'avérer utiles à des opérateurs se trouvant dans des situations analogues. Nous nous bornons ici à relever trois points majeurs.

\section{$\square$ Intégrer la dimension « urgence » dans le développement}

Mieux vaut intégrer la dimension «urgence» dans un travail permanent de développement que d'envisager un passage de l'urgence au développement. Les efforts d'une communauté locale pour améliorer sa situation ne s'arrêtent pas lorsque surgit une crise. Certes, la situation se fait plus complexe et les difficultés augmentent, mais les populations sinistrées démontrent la volonté, et la capacité, de s'en sortir par leurs propres moyens. Si une organisation d'aide venant de l'extérieur ne tient pas compte de ce principe de base et offre une aide non adéquate, elle se met en porte-à-faux dans la perspective du développement local qu'elle risque de freiner et de retarder. Insister sur les capacités locales de résoudre la crise part aussi de l'idée que les communautés de base (sinistrées et non sinistrées) participent, dans la mesure du possible, à la gestion de la crise et à la recherche de solutions aux situations d'urgence.

Lorsque la crise a éclaté au Burundi, la plupart des organisations de développement ont abandonné leurs activités, principalement à cause de la sécurité de leur personnel. L'OAP a choisi de rester et d'intégrer l'aide humanitaire dans son approche d'appui au développement. Ainsi elle a pu continuer à collaborer avec ses partenaires habituels (les résidents), à définir avec eux les nouveaux besoins et à les aider à accueillir les personnes déplacées venues dans leur commune. L'intégration d'activités liées à la crise (l'appui aux sinistrés) dans le programme régulier a nécessité la mobilisation non seulement des personnes déplacées, mais de la population dans son ensemble, ainsi que l'organisation de séances de sensibilisation afin d'amener les partenaires de l'OAP à devenir partie prenante dans la restructuration de leur société. La création des commissions communales découle de cette préoccupation.

La mobilisation de la population a permis, outre de trouver des solutions locales aux problèmes d'urgence, de réunir différents groupes de personnes qui d'ordinaire ne se rencontrent pas et qui ont pu ainsi discuter ensemble, partager des soucis d'intérêt général et réfléchir afin d'élaborer de concert des solutions aux problèmes communs. Plus concrètement, cela a été le cas pour plusieurs jeunes de Bujumbura-Rural qui se sont mobilisés autour de l'OAP à travers les activités de l'artisanat, l'agriculture, la distribution de vivres, etc., et qui jouent un rôle important dans la vie sociale locale. En outre, il est probable que ce genre de rencontre contribue à la réconciliation et a un effet de pacification. 
Pour suivre cette stratégie, l'OAP bénéficie de certains atouts:

口 une conception claire et explicite quant à son approche et sa philosophie d'intervention;

๖ une bonne connaissance du milieu, spécialement de la mentalité des populations de Bujumbura-Rural ;

- la confiance de la population: partenaire habituel, l'OAP n'a pas eu de problèmes à se faire accepter au moment de la crise ;

- une politique d'information transparente permettant d'entretenir, entre autres, des relations directes avec les différents acteurs concernés, et notamment avec les autorités locales, tant provinciales que communales;

口 une position claire par rapport à son propre rôle: elle évite à tout prix la substitution, c'est-à-dire d'agir à la place des opérateurs locaux (commissions communales, autorités, etc.) ; si ceux-ci ne développent pas d'initiatives, ce n'est pas à l'OAP d'agir;

口 un fort engagement personnel de l'équipe (entièrement burundaise).

La présence d'organisations de développement dans des moments de crise est très importante, à condition cependant que certains critères soient remplis, comme ceux cités ci-dessus. De plus, dans le travail opérationnel, il y a lieu de bien distinguer entre ce qui relève du programme régulier d'appui au développement local et ce qui fait partie d'activités de secours et d'aide d'urgence. Cette distinction est capitale pour ne pas saboter un aspect au détriment de l'autre et assurer la cohérence. Il faut accorder une grande importance à l'analyse précise de toutes les situations avant d'intervenir et à l'évaluation permanente. L'OAP a fait l'expérience que seul un bon système d'identification et de suivi-évaluation permettait d'assurer cette articulation difficile mais nécessaire.

Dès le début de la crise du Burundi, les besoins qui ont été exprimés à l'OAP à titre d'aide d'urgence étaient les suivants: nourriture, soins, matériel aratoire, matériel de couchage, semences, scolarisation des enfants, ustensiles de cuisine, vêtements. L'OAP s'est attelée à rassembler tous ces biens afin de pouvoir intervenir très rapidement, sur la base d'une analyse approfondie des besoins et des capacités locales.

Les distributions étaient toujours précédées de séances d'animation pour faire comprendre aux bénéficiaires que personne ne pourrait intervenir de manière indéfinie, qu'aucune organisation ne pourrait se substituer à leurs propres efforts, et qu'ils devaient viser l'auto-prise en charge. A titre d'illustration, le matériel de couchage distribué a été commandé chez les personnes déplacées pratiquant ce même métier. Cette approche a également permis la fusion des personnes déplacées et non déplacées, des deux ethnies. Les menuisiers déplacés ont continué à valoriser leur métier en fabriquant le mobilier scolaire; les maçons et les charpentiers ont réparé les infrastructures publiques endommagées par la crise. Tous ces artisans ont bénéficié des marchés de l'OAP et ont pu se réinstaller. L'OAP a également accordé plus d'importance à la distribution du matériel aratoire et des semences de sorte que les agriculteurs déplacés se sont rapidement remis au travail.

Certains besoins, bien que très importants, n'étaient pas du ressort de l'OAP; il s'agissait principalement de la scolarisation des orphelins et des besoins 
sanitaires. Après analyse, l'OAP a décidé de développer une synergie avec d'autres organisations, dans laquelle elle servait de relais en fournissant du matériel d'hygiène pour les personnes déplacées et des cahiers pour les orphelins.

L'OAP a collaboré avec plusieurs organisations pour atteindre ses objectifs. Certains besoins financiers ont été honorés par d'autres organisations comme le PNUD (Programme des Nations Unies pour le développement), Twitezimbere (ONG nationale soutenue par des organisations financières multilatérales telles la Banque mondiale, la Banque africaine de développement, etc.), Terre des hommes, INADES (Institut africain pour le développement économique et social), PREFED (Programme régional de formation et d'échanges pour le développement), agences de coopération étrangères... Cette méthodologie a permis aux partenaires de terrain d'une part de s'ouvrir à d'autres bailleurs, évitant ainsi que les bénéficiaires ne deviennent dépendants que d'une seule organisation, et d'autre part de comparer la philosophie des différents intervenants.

L'OAP ayant toujours comme objectif d'amener la population à l'idée de l'autoprise en charge, elle travaille avec des groupes qui partagent ce point de vue et qui manifestent clairement leur envie de quitter le plus rapidement possible la situation de dépendance pour acquérir leur autonomie dans les meilleurs délais. Cependant, l'OAP n'a jamais essayé d'empêcher certains bénéficiaires qui le souhaitaient de collaborer avec des organisations d'aide pratiquant plutôt une aide de type assistanat; dans ces cas, l'OAP se retire, tout en expliquant les raisons de son retrait.

Cela dit, la coexistence de multiples organisations d'aide aux démarches différentes est un facteur négatif pour le développement local, mais auquel on ne peut guère remédier sous peine d'imposer la philosophie d'une organisation d'aide. L'OAP est très active dans les mesures de concertation et d'échange entre les responsables des organisations car seules ces mesures peuvent conduire à une amélioration de la collaboration.

Dans sa philosophie d'aide humanitaire, l'OAP n'a par exemple jamais distribué gratuitement les semences, mais les fournissait à crédit aux bénéficiaires afin d'assurer le renouvellement du stock des semences pour les saisons suivantes. Cette philosophie, comprise, acceptée et appréciée par les autorités locales et les bénéficiaires, n'était pas partagée par tous les autres intervenants. Dans sa promotion de la politique de petit crédit, l'OAP n'a pas été comprise par des organisations qui pratiquaient le «don financier». Trop souvent, les organisations classiques d'aide humanitaire s'occupent généralement de la distribution gratuite sans pour autant se demander ce que va devenir la population à la fin des interventions.

Devant cette divergence, il revient à l'organisation d'analyser chaque situation et de la faire comprendre aux partenaires sur le terrain. Lorsqu'une organisation de développement doit faire face à ces situations, des séances régulières d'évaluation s'imposent pour mieux cerner les origines des problèmes, faire le bilan des différentes expériences et créer la base pour piloter les actions dans le futur. Ainsi on peut rectifier le tir si cela s'avère nécessaire. L'OAP a régulièrement tenu des séances d'évaluation sous forme d'ateliers, de réunions, de séminaires de réflexion, de réunions d'écoute des bénéficiaires. Grâce à ces séances, elle a 
décidé après, six mois, de diminuer les activités d'aide humanitaire et de continuer son programme d'appui au développement sous forme d'activités de relance économique et de rénovation de l'habitat rural, tout en continuant à intervenir de manière ponctuelle en aide d'urgence.

En conclusion, dans une situation de crise les besoins sont multiples, les moyens limités et la population déstabilisée. Le programme d'aide humanitaire court alors le risque de dominer toutes les activités au détriment de l'objectif d'autopromotion. C'est pourquoi une analyse précise de chaque situation s'avère importante avant d'intervenir: parmi les multitudes des besoins exprimés par la population, il y a lieu de déterminer avec exactitude ceux qui relèvent de l'humanitaire et ceux qui favorisent l'autopromotion. Cependant, l'aide humanitaire peut être valorisée et jouer un rôle très important à condition qu'elle soit intégrée - dès le début - dans une philosophie d'autosuffisance.

\section{$\square$ Transparence, dialogue permanent et neutralité}

Une organisation d'appui au développement local comme l'OAP peut jouer un rôle important pour appuyer la reconstruction de la société dans une situation de crise, à condition qu'elle soit acceptée par les principaux groupes d'acteurs et qu'elle reste politiquement neutre. Or, les situations de crise sont toujours caractérisées par d'importants conflits d'intérêts entre les différents acteurs et il est alors difficile de défendre une ligne cohérente, de résister aux pressions et de respecter les principes établis.

Afin de pouvoir jouer son rôle d'appui aux personnes déplacées tout en se situant clairement dans une perspective de développement local, l'OAP attache une grande importance aux "relations extérieures» : elle entretient des contacts et des échanges réguliers avec tous les acteurs et travaille en réseau; elle mène une politique d'information claire, transparente et uniforme sur son travail et les buts poursuivis; en collaborant avec tous les acteurs qui remplissent les critères de l'organisation et se conforment aux décisions des structures représentant les intérêts des populations (commissions communales), elle confirme sa neutralité; enfin, pour obtenir la confiance des différents interlocuteurs, elle accorde une grande importance aux relations publiques et à la diplomatie.

L'OAP doit sa crédibilité à une politique constante par rapport au contenu de ses messages, aux stratégies d'information, aux approches et aux pratiques sur le terrain. Tous les membres de l'OAP partagent la même philosophie et tiennent le même langage. La question ethnique n'intervient ni dans la composition de l'équipe, ni dans le choix des communes ou des partenaires. Ici aussi, il est pratiqué une stricte neutralité.

La transparence et le dialogue permanent jouent notamment à trois niveaux :

- L'administration locale - L'équipe opérationnelle de l'OAP a toujours informé les autorités locales (administrateurs et gouverneur de la province) sur les programmes de travail et sur tous ses déplacements. En contrepartie, chaque fois qu'une zone était dans l'insécurité, les autorités locales informaient l'équipe qui devait en tenir compte dans la programmation de ses activités. Grâce à ce dialogue, les services chargés de la sécurité étaient sensibilisés par les autorités locales à la protection des infrastructures rénovées par l'OAP (écoles en construction, groupements d'élevage...). Ainsi par 
exemple, sur dix groupements d'élevage de poules soutenus par l'OAP, deux seuls ont été pillés par des éléments armés.

L'OAP invite toujours les représentants de l'autorité locale à participer aux travaux de la commission communale; cette participation est primordiale, non seulement parce que l'autorité locale a des tâches à remplir qui relèvent de sa responsabilité et de sa compétence, mais aussi pour améliorer le contact et les relations de confiance entre «administrateurs » et «administrés».

L'OAP porte une grande attention au rôle de médiation entre des acteurs ayant des intérêts bien distincts, mais qui se trouvent obligés de collaborer car ils vivent sur le même territoire. Pour des raisons historiques (dictature militaire), il y a encore des progrès à accomplir pour arriver à une gestion commune ou démocratique des problèmes d'intérêt général. Une organisation de développement comme l'OAP peut contribuer à ce processus en mettant les acteurs en relation et en favorisant les échanges et la recherche des solutions. Cet axe de travail de type «renforcement institutionnel» est autant prioritaire que les appuis matériels fournis pour améliorer la situation socioéconomique des populations.

- Les partenaires de terrain (groupements, associations, promoteurs individuels, etc.) passent régulièrement pour s'informer du programme et des activités; la confiance est ainsi établie entre les différents interlocuteurs. L'équipe de l'OAP consacre régulièrement une journée à recevoir et à écouter les représentants des populations, et cette journée est respectée même pendant la période de congé de l'équipe. Dès qu'apparaît une situation de conflit dans une zone, ces interlocuteurs informent le personnel de l'OAP et l'aident à ajuster le calendrier des visites de terrain. Dans ce contexte, ces mêmes interlocuteurs, à titre bénévole, assurent la liaison entre l'OAP, les structures relais et les bénéficiaires de l'appui, ainsi que le suivi et le recouvrement.

- Les commissions communales et les associations relais - Afin de pouvoir jouer pleinement son rôle d'appui et de permettre aux acteurs locaux d'assumer leurs responsabilités, l'OAP a dû prévoir des structures relais au niveau des communes. En accord avec la population, elle a fait un travail intense d'animation en janvier 1995 pour expliquer la nécessité de la formation d'une commission communale chargée de

- servir d'interface entre la population et l'OAP;

- sensibiliser la population à l'importance des projets de développement malgré la crise;

- sensibiliser la population à l'importance de former des groupements ;

- aider les soumissionnaires à remplir les fiches de projet et les dossiers;

- assurer l'information sur ce qui se fait et ce qui est prévu;

- choisir les critères de priorité des projets;

- analyser et trier les projets;

- défendre les projets auprès de l'OAP;

- suivre l'exécution du projet dans les différentes phases;

- assurer le recouvrement;

- appuyer techniquement les artisans dans la mesure de ses possibilités. 
Les commissions sont composées d'un représentant de l'autorité locale, des techniciens communaux, des sages de la commune, du représentant de l'Eglise, des délégués des associations, des représentants des personnes déplacées, etc. Comme mentionné plus haut, en l'absence de commissions communales, ce sont des associations relais (choisies parmi des associations existantes) qui sont les interlocuteurs de l'OAP.

La formation et l'installation de ces structures décentralisées ont permis à l'équipe opérationnelle non seulement de diminuer les descentes sur le terrain et de courir moins de risques, mais surtout de responsabiliser les différents types d'acteurs de la commune pour trouver, à leur niveau, des solutions aux problèmes d'intérêt général.

\section{$\square$ Régler les questions institutionnelles de fond}

Pour la mise en œuvre durable d'une approche comme celle pratiquée par l'OAP, il y a des questions institutionnelles de fond qui doivent être réglées relatives à la taille et à la composition de l'équipe, à son degré d'autonomie, etc.

Trop d'organisations impliquées dans le développement local ont une vie courte. Or, le changement social d'un pays comme le Burundi, comprenant le développement socio-économique, la consolidation politique et aussi la gestion de crises et de situations d'urgence, nécessite des institutions solides, ayant une certaine éthique et une vision claire de l'évolution de la société. C'est dans cette optique que l'OAP réfléchit aux différentes questions concernant la durabilité de son organisation, sa taille, la composition de son équipe, son rayon d'action, son autonomie financière, etc.

Une organisation comme l'OAP est tôt ou tard confrontée à la question de la taille. En effet, suite à diverses sollicitations, aux offres de collaboration, aux demandes d'appui, elle a une tendance naturelle à s'agrandir. Cependant, l'OAP est arrivée à la conclusion qu'il est préférable de garder une taille modeste ${ }^{7}$ pour bien maîtriser tous les paramètres, dont les principaux, jugés essentiels pour travailler dans une situation de crise, sont l'engagement intègre de chaque membre de l'équipe, le maintien d'une certaine cohésion et une situation de confiance. Il est primordial que chaque membre partage la même «lecture» des problèmes et le même engagement personnel. L'expérience de l'OAP prouve que plus la taille est modeste, plus la coordination interne, les échanges, la recherche de solutions, etc., sont facilités. Cette limitation de l'équipe responsable à un certain nombre de professionnels n'empêche d'ailleurs en rien l'ouverture vers des collaborations avec d'autres organisations et des mandataires pour chercher de nouvelles synergies, compléter les ressources manquantes, créer des alliances...

Le maintien d'une équipe modeste constitue aussi une pression permanente pour diminuer le risque de se substituer aux acteurs locaux. La création des structures relais au niveau communal est certes due à une certaine philosophie de développement, mais elle s'impose également du fait que l'OAP n'a pas les capacités humaines pour remplir les tâches assignées à ces structures.

Pour faire un travail dans la continuité, une organisation comme l'OAP doit aussi penser à sa propre autonomie. L'idée n'est pas qu'une organisation

7. Voir note 4 pour la composition de l'équipe. 
travaillant dans des situations de crise avec une optique de promotion du développement local puisse devenir un jour autonome; il est par contre souhaitable qu'elle évolue vers une situation organisationnelle et financière qui lui donne une certaine marge de manœuvre et de liberté d'action et la mette à l'abri de changements inattendus dans les relations de partenariat en cours (dus par exemple à des changements d'orientation chez les bailleurs de fonds).

Deux voies sont actuellement mises en œuvre - en parallèle - pour avancer dans cette réflexion. La première consiste à diversifier les sources de financement ${ }^{8}$ pour éviter la dépendance d'un seul partenaire financier; la seconde consiste à augmenter les revenus propres pour des services rendus à des partenaires de l'OAP qui réalisent des projets à caractère économique. Outre les groupements d'artisans et de cultivateurs formés par les résidents, on compte des associations de personnes déplacées ou de victimes de la crise, telles les associations des veuves, qui ont des projets productifs pour lesquels l'OAP a accordé des crédits. Elles ont des intérêts à payer et les prêts sont remboursables. Ces intérêts constituent une contribution pour couvrir les frais d'encadrement et de suivi.

\section{CONCLUSION}

L'exemple de l'intervention de l'OAP au Burundi ne prétend pas être un modèle idéal à imiter tel quel. Il veut simplement illustrer l'application sur le terrain d'un certain nombre de principes qui constituent une tentative pour résoudre les problèmes liés à l'articulation entre aide au développement et aide d'urgence. Ces principes sont d'ailleurs défendus par plusieurs organisations et figurent dans leurs déclarations d'intention, mais leur application dans le travail pratique journalier fait en règle général défaut.

Le travail de l'OAP ne se fait pas sans difficultés; elle est confrontée à une situation politique difficile, à l'insécurité et à la violence, à la méfiance, au désespoir, au mépris de l'intérêt général en faveur d'intérêts personnels, etc. Mais ces éléments ne sont pas spécifiquement typiques du Burundi; on les retrouve pratiquement dans tous les contextes de crise. L'important est de savoir comment une organisation d'appui comme l'OAP tient compte de la spécificité de ces facteurs dans un contexte donné, quels instruments et quelles méthodes de travail elle choisit, comment elle s'organise, afin de pouvoir faire un travail qui contribue finalement d'une manière durable à la solution des problèmes.

L'exemple de l'OAP veut aussi être un témoignage d'espoir: ce n'est pas parce que la situation est particulièrement difficile dans un pays que le système d'aide doit arrêter son appui. Le cas de l'OAP montre que, tout en respectant les règles de sécurité, il existe une marge de manœuvre importante pour une organisation de développement moyennant le respect strict de certains principes et l'utilisation d'instruments et de méthodes de travail adéquats.

8. L'OAP a pu intéresser une ONG française (le Centre d'appui aux initiatives locales CIEPAC), qui a été d'accord de négocier sa contribution, en fonction du programme établi par l'OAP, pour la partie du budget couverte ni par les subventions de l'IUED et de la Division de l'aide humanitaire, ni par les revenus propres. 